\title{
Flutter control of a two-degrees-of-freedom airfoil using a nonlinear tuned vibration absorber
}

\author{
Arnaud Malher ${ }^{\mathrm{a}}$, Cyril Touzéa ${ }^{\mathrm{a}}$, Olivier Doaréa ${ }^{\mathrm{a}}$, Giuseppe Habib ${ }^{\mathrm{b}}$, Gaëtan Kerschen ${ }^{\mathrm{b}}$ \\ ${ }^{a}$ IMSIA (Institute of Mechanical Sciences and Industrial Applications), \\ ENSTA ParisTech - CNRS - EDF - CEA, \\ Université Paris-Saclay, \\ 828 Bd des Maréchaux, 91732 Palaiseau Cedex, France \\ ${ }^{b}$ Space Structures and Systems Laboratory \\ Department of Aerospace and Mechanical Engineering \\ University of Liège, Belgium
}

\begin{abstract}
The influence of a Nonlinear Tuned Vibration Absorber (NLTVA) on the airfoil flutter is investigated. In particular, its effect on the instability threshold and the potential subcriticality of the bifurcation is analyzed. For that purpose, the airfoil is modeled using the classical pitch and plunge aeroelastic model together with a linear approach for the aerodynamic loads. Large amplitude motions of the airfoil are taken into account with nonlinear restoring forces for the pitch and plunge degrees of freedom. The two cases of a hardening and a softening spring behavior are investigated. The influence of each NLTVA parameter is studied and an optimum tuning of these parameters is found. The study reveals the ability of the NLTVA to shift the instability, avoid its possible subcriticality and reduce the LCOs amplitude.
\end{abstract}

Keywords: Flutter instability, Nonlinear tuned vibration absorber, Tuned mass damper, passive control

\section{Introduction}

When slender structures, such as bridge deck or airfoil, undergo wind excitation, a flutter instability, triggered by a Hopf bifurcation, may arise. The flow velocity for which the instability starts is called flutter velocity. This phenomenon is detrimental and may even lead to fatal vibrations of the structure.

Numerous strategies have been investigated in the past in order to control the flutter instability. Most of them concern active control techniques, such as governing a trailing edge flap on the wing $[1,2,3]$ or using piezoelectric actuators [4]. The reader may refer to the Dowell's book on aeroelasticity [5] for more details on flutter active control. The alternative strategy is the use of passive control techniques, which is also the aim of the present study. This research topic is relatively recent. Most efforts have been devoted to bridge deck passive control $[6,7,8]$.

Email address: arnaud.malher@ensta-paristech.fr (Arnaud Malher) 
The most classical passive device for controlling flutter instabilities is the tuned vibration absorber initially developed by Den Hartog [9], and generally known as TMD (Tuned Mass Damper) or TVA (Tuned Vibration absorber). This device consists of a small lumped mass attached to the primary structure through a linear spring and a damper. If its eigenfrequency and damping ratio are correctly tuned, it can significantly shift the flutter speed [10]. Nevertheless, the TMD has no effect on the nonlinearities arising in the post-critical regime.

Lee et al. $[11,12]$ proposed to use a nonlinear energy sink (NES) to control the flutter instability. The NES has been initially investigated in the field of vibration control $[13,14,15]$, it consists of an oscillator linked to the primary structure through an essential nonlinear stiffness (i.e. with no linear part), leading to targeted energy transfer [16]. Applied to the flutter control, the method shows an important reduction in the amplitude of the LCOs $[11,12]$. Nevertheless, it has no effect on the flutter velocity, and needs a certain amount of energy to be activated.

In the present study, the use of a nonlinear tuned vibration absorber (NLTVA), as proposed by Viguié and Kerschen [17] and Habib et al [18] is investigated. The distinctive feature of the NLTVA is that it possesses both a linear and a nonlinear stiffness. The linear part of the absorber enables to recover the optimal control given by a TMD, whereas its nonlinear part can be tuned in order to enhance the control of the primary system nonlinearities, using a so-called principle of similarity [19]. Its effectiveness has been proven on a Van der Pol-Duffing oscillator [20]. Moreover, several experimental realizations of the NLTVA have been recently explored [21, 22] and a tuning rule for the NLTVA parameters coupled with a primary system having polynomial nonlinearities has been proposed using the Den Hartog's equal peak method [19].

In the present context of the flutter instability, two main design rules are followed for optimizing the NLTVA characteristics. First, the linear stiffness of the absorber is tuned in order to increase as much as possible the critical flutter velocity, thus repelling the appearance of LCO to larger flow velocities. This step consists in finding the optimal values of a TMD in order to control the flutter instability, for which preliminary results can be found in [23] for a specific set of airfoil parameters. Secondly, the nonlinear stiffness of the NLTVA may be tuned in order to enforce supercriticality, as well as to reduce the LCO amplitudes. The aim of the present study is thus to extend the results presented in [20], obtained for a Van der Pol-Duffing oscillator as primary structure. To get closer to a real airfoil situation, the primary structure is here selected as the classical two degrees-of-freedom aeroelastic system, with pitch and plunge motions as generalized coordinates [5]. The quasi-steady theory is used to estimate the aerodynamic loads. In order to take into account the nonlinear structural behavior for large displacements, the model includes cubic stiffness terms in the pitch and plunge motion. This allows the system to develop LCO in the post-critical regime.

The first section is devoted to the linear stability analysis. The optimization of the linear characteristics of the vibration absorber is investigated, showing in particular how the flutter velocity can be repelled to larger values using a well-tuned absorber. The airfoil parameters are then varied and a design rule is proposed in order to find easily the optimized linear values. Then the influence of the nonlinear part of the NLTVA is investigated. Two different cases are specifically studied for the airfoil nonlinear restoring force: a hardening and a softening scenario. These results give a number of fruitful strategies in order to control passively the flutter instability by means of a nonlinear vibration absorber. 


\section{Equations of motion}

\subsection{Model equations}

The classical pitch and plunge model is used to describe the airfoil motion, see e.g. [5]. Pitch and plunge are respectively described by the heave $h$ and the angle of attack $\alpha$ as shown in Fig. 1 . The geometrical parameters of the airfoil are the chord $c$, the semi-chord $b$ and the lifting surface $S$. EC is the elastic center, GC the gravity center and AC the aerodynamic center. The distance between $\mathrm{AC}$ and $\mathrm{EC}$ is denoted by $e$. The inertia terms of the airfoil are the mass $M$, the inertia moment $I_{\alpha}$ and the static moment $S_{\alpha}$. The static moment $S_{\alpha}$ is equal to $M x_{C G}$, where $x_{C G}$ is the distance between EC and GC. The pitch mode is described by the stiffness $k_{\alpha}$ and the damping $c_{\alpha}$ and the plunge motion by the stiffness $k_{h}$ and the damping $c_{h}$. The model encompasses structural nonlinearities, which have the beneficial effect of limiting the dynamics to LCOs in the post-critical regime. Nonlinear terms in the plunge and pitch stiffnesses, respectively $k_{h 3}$ and $k_{\alpha 3}$, are thus introduced in a similar fashion as in $[11,12,24,25]$ in order to take into account the potential nonlinearities of the aeroelastic system that can arise from e.g. geometric or localized nonlinearities.

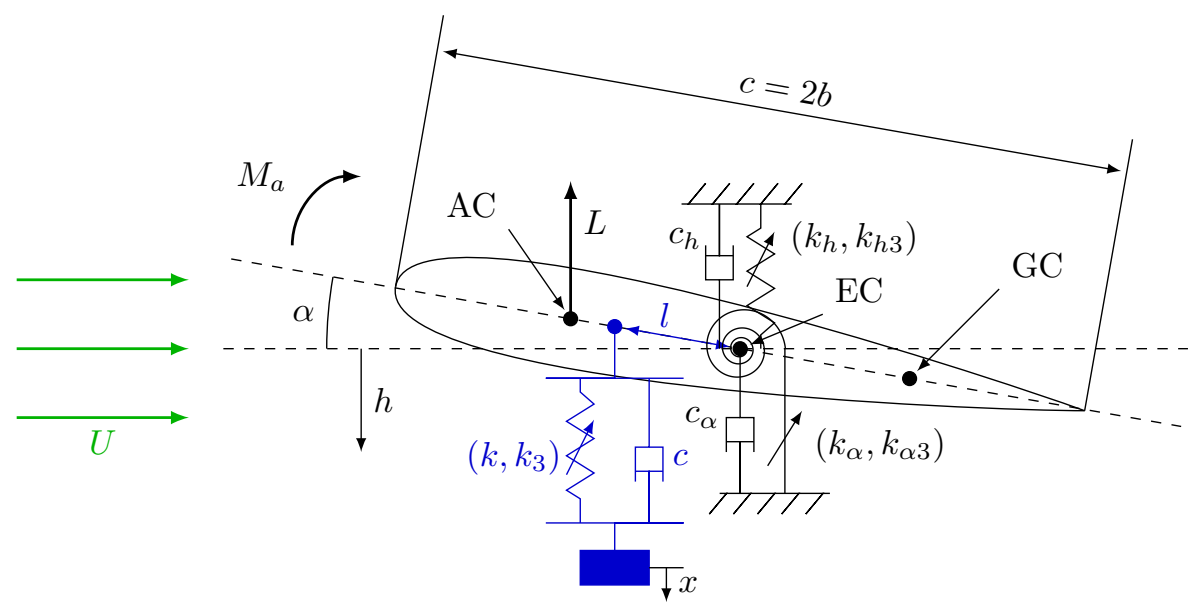

Figure 1: Sketch of the two degrees of freedom airfoil (main structure) in black coupled with the NLTVA (absorber) in blue.

The flow goes from left to right with velocity $U$, as illustrated in Fig. 1. It produces two aerodynamic loads, the lift $L$ and the aerodynamic moment $M_{a}$. The quasi-steady theory is selected for modeling them, see e.g. $[5,26]$. It gives the following expressions : $L=(1 / 2) \rho S U^{2} \mathrm{~d} C_{l}(\alpha+\dot{h} / U)$ and $M_{a}=(1 / 2) \rho S c U^{2} e \mathrm{~d} C_{l}(\alpha+\dot{h} / U)$, where $\rho$ is the fluid density and $\mathrm{d} C_{l}$ is the slope at zero angle of attack of the lift coefficient.

The NLTVA is attached along the mid-chord of the airfoil at distance $l$ from EC. It is composed of a mass $m$, a spring of linear stiffness $k$ and cubic stiffness $k_{3}$, and a dashpot of damping $c$. Finally, the equations of motion read : 


$$
\begin{array}{r}
M \ddot{h}+S_{\alpha} \ddot{\alpha}+\left(c_{h}+B U\right) \dot{h}+c(\dot{h}-\dot{x}-l \dot{\alpha})+k_{h} h+B U^{2} \alpha+k(h-x-l \alpha) \\
+k_{h 3} h^{3}+k_{3}(h-x-l \alpha)^{3}=0, \\
I_{\alpha} \ddot{\alpha}+S_{\alpha} \ddot{h}+c_{\alpha} \dot{\alpha}+c l(\dot{x}+l \dot{\alpha}-\dot{h})-N U \dot{h}+\left(k_{\alpha}-N U^{2}\right) \alpha+k l(x+l \alpha-h) \\
+k_{\alpha 3} \alpha^{3}+k_{3} l(x+l \alpha-h)^{3}=0, \\
m \ddot{x}+c(\dot{x}+l \dot{\alpha}-\dot{h})+k(x+l \alpha-h)+k_{3}(x+l \alpha-h)^{3}=0,
\end{array}
$$

where $B=(1 / 2) \rho S \mathrm{~d} C_{l}$ and $N=(1 / 2) \rho S e \mathrm{~d} C_{l}$.

The aim of the linear part of the NLTVA, which can be seen as a TMD, is to repel the flutter velocity. The goal of its nonlinear part is to reduce the LCO amplitude and ensure the supercriticality of the bifurcation caused by the flutter instability. The NLTVA nonlinear part is limited to a cubic stiffness in order to have the same functional form as the airfoil nonlinearity as prescribed in $[20]$.

In order to reduce the number of the system parameters, the equations of motion are made dimensionless, by introducing

$$
y=h / b \quad \text { and } \quad \tilde{x}=x / b .
$$

The inertia coefficients become

$$
r_{\alpha}=\sqrt{I_{\alpha} / M b^{2}} \text { and } \quad x_{\alpha}=S_{\alpha} / M b
$$

Afterward, by introducing the dimensionless time $\tau=\omega_{\alpha} t$, where $\omega_{\alpha}=\sqrt{k_{\alpha} / I_{\alpha}}$, the expression of the frequency ratio, dimensionless cubic stiffnesses and damping ratios are obtained as,

$\omega_{h}=\sqrt{k_{h} / M}, \Omega=\omega_{h} / \omega_{\alpha}, \xi_{h}=k_{h 3} b^{2} / M \omega_{\alpha}^{2}, \xi_{\alpha}=k_{\alpha 3} / M b^{2} \omega_{\alpha}^{2}, \zeta_{h}=c_{h} / M \omega_{\alpha}$ and $\zeta_{\alpha}=c_{\alpha} / M b^{2} \omega_{\alpha}$.

Then the dimensionless aerodynamic parameters are introduced as follows

$$
\tilde{U}=U / b \omega_{\alpha}, \beta=B b / M \text { and } \nu=N / M .
$$

Eventually, the dimensionless NLTVA parameters are

$$
\varepsilon=m / M, \omega=\sqrt{k / m}, \gamma=\omega^{2} / \omega_{\alpha}^{2}, \lambda=l / b, \zeta=c / m \omega_{\alpha} \text { and } \xi=k_{3} b^{2} / m \omega_{\alpha}^{2} .
$$

The equations of motion finally can be recast in compact form as :

$$
\mathbf{M q} \mathbf{q}^{\prime \prime}+\mathbf{C q}^{\prime}+\mathbf{K q}+\mathbf{F}(\mathbf{q})=\mathbf{0}
$$

with

$$
\begin{aligned}
& \mathbf{q}=\left[\begin{array}{l}
y \\
\alpha \\
\tilde{x}
\end{array}\right], \quad \mathbf{M}=\left[\begin{array}{ccc}
1 & x_{\alpha} & 0 \\
x_{\alpha} & r_{\alpha}^{2} & 0 \\
0 & 0 & 1
\end{array}\right], \quad \mathbf{C}=\left[\begin{array}{ccc}
\zeta_{h}+\varepsilon \zeta+\beta \tilde{U} & -\varepsilon \zeta \lambda & -\varepsilon \zeta \\
-\nu \tilde{U}-\varepsilon \zeta \lambda & \zeta_{\alpha}+\varepsilon \zeta \lambda^{2} & \varepsilon \zeta \lambda \\
-\zeta & \zeta \lambda & \zeta
\end{array}\right], \\
& \mathbf{K}=\left[\begin{array}{ccc}
\Omega^{2}+\varepsilon \gamma & \beta \tilde{U}^{2}-\varepsilon \gamma \lambda & -\varepsilon \gamma \\
-\varepsilon \gamma \lambda & r_{\alpha}^{2}-\nu \tilde{U}^{2}+\varepsilon \gamma \lambda^{2} & \varepsilon \gamma \lambda \\
-\gamma & \gamma \lambda & \gamma
\end{array}\right] \quad \text { and } \quad \mathbf{F}(\mathbf{q})=\left[\begin{array}{c}
\xi_{h} y^{3}+\epsilon \xi(y-\tilde{x}-\lambda \alpha)^{3} \\
\xi_{\alpha} \alpha^{3}+\epsilon \lambda \xi(\tilde{x}+\lambda \alpha-y)^{3} \\
\xi(\tilde{x}+\lambda \alpha-y)^{3}
\end{array}\right],
\end{aligned}
$$


where ()$^{\prime}=d / d \tau$.

The characteristics of the NLTVA are defined according the following five parameters:

1. the mass ratio $\varepsilon=m / M$, between the mass of the NLTVA and that of the wing;

2. the dimensionless distance $\lambda$ that specifies the location where the NLTVA is attached;

3. the NLTVA reduced eigenfrequency $\gamma=\omega^{2} / \omega_{\alpha}^{2}$, characterizing the eigenfrequency of the NLTVA compared to that of pitch motion;

4. the NLTVA damping ratio $\zeta=c / m \omega_{\alpha}$;

5. the NLTVA reduced cubic stiffness $\xi=k_{3} /\left(m \omega_{\alpha}^{2}\right)$.

For the present study, the mass ratio has been taken equal to $5 \%$. The larger $\varepsilon$ the more efficient the NLTVA will be, however one must keep in mind that the absorber needs to have a negligible mass as compared to the airfoil. In this work, the NLTVA is located at the leading edge of the profile, thus $\lambda=1$. Note that its effect has been found, in our study, to be directly proportional to $\lambda$. Hence the location where its influence on the airfoil is optimal has been selected. Eventually, $\gamma$ and $\zeta$ are thus selected as our linear control parameters and $\xi$ as our nonlinear control parameter, the optimal tuning of which is searched for.

\subsection{Flutter velocity}

The aim of this section is to introduce the flutter velocity of the airfoil system alone, which will be afterward compared to that obtained with the presence of the absorber. The linear part of the equations of motion without the NLTVA writes

$$
\left[\begin{array}{cc}
1 & x_{\alpha} \\
x_{\alpha} & r_{\alpha}^{2}
\end{array}\right]\left[\begin{array}{c}
y^{\prime \prime} \\
\alpha^{\prime \prime}
\end{array}\right]+\left[\begin{array}{cc}
\zeta_{h}+\beta \tilde{U} & 0 \\
-\nu \tilde{U} & \zeta_{\alpha}
\end{array}\right]\left[\begin{array}{c}
y^{\prime} \\
\alpha^{\prime}
\end{array}\right]+\left[\begin{array}{cc}
\Omega^{2} & \beta \tilde{U}^{2} \\
0 & r_{\alpha}^{2}-\nu \tilde{U}^{2}
\end{array}\right]\left[\begin{array}{l}
y \\
\alpha
\end{array}\right]=\mathbf{0}
$$

We assume a solution of the form

$$
\left[\begin{array}{l}
y \\
\alpha
\end{array}\right]=\left[\begin{array}{l}
y_{0} \\
\alpha_{0}
\end{array}\right] \exp (z t)
$$

where $y_{0}=y(0), \alpha_{0}=\alpha(0)$ and $z \in \mathbb{C}$. Therefore, three situations are possible,

1. $\Re(z)<0$, the airfoil motion is stable,

2. $\Re(z) \geq 0$ and $\Im(z) \neq 0$, the airfoil encounters flutter,

3. $\Re(z) \geq 0$ and $\Im(z)=0$, the airfoil encounters divergence.

The linear system Eq. (3) with the ansatz (4) writes

$$
\mathbf{B}\left[\begin{array}{l}
y_{0} \\
\alpha_{0}
\end{array}\right] \exp (z t)=\mathbf{0} \quad \text { with } \quad \mathbf{B}=\left[\begin{array}{cc}
z^{2}+\zeta_{h} z+\beta \tilde{U} z+\Omega^{2} & x_{\alpha} z^{2}+\beta \tilde{U}^{2} \\
x_{\alpha} z^{2}-\nu \tilde{U} z & r_{\alpha}^{2} z^{2}+\zeta_{\alpha} z+r_{\alpha}^{2}-\nu \tilde{U}^{2}
\end{array}\right]
$$

By canceling the determinant of $\mathbf{B}$, the flutter velocity $\tilde{U}_{f}$ can be found, as shown in $[5,26,27$, $28,29]$.

The next section is devoted to the linear stability analysis in the presence of the NLTVA. The calculation will follow the same guidelines recalled here. 


\section{Linear stability analysis}

\subsection{Optimization of the NLTVA linear parameters.}

The aim of this section is to study the influence of the design parameters $\gamma$ and $\zeta$ on the flutter velocity $\tilde{U}_{f}$. The targeted optimal values are those for which $\tilde{U}_{f}$ is as large as possible, in order to repel the instability.

In the remaining of this study, unless explicitly mentioned, the airfoil linear parameters of Eq. (2) are equal to the parameters from [5]. They are gathered in Table 1 and define the reference case. In the next section, the airfoil parameters will be varied in order to investigate their influence on the NLTVA tuning and effectiveness. In this section, the nonlinear terms are not taken into account.

\begin{tabular}{ccccccc}
\hline$x_{\alpha}$ & $r_{\alpha}$ & $\beta$ & $\nu$ & $\Omega$ & $\zeta_{\alpha}$ & $\zeta_{h}$ \\
\hline 0.2 & 0.5 & 0.2 & 0.08 & 0.5 & 0.01 & 0.01 \\
\hline
\end{tabular}

Table 1: Dimensionless aeroelastic parameters of the reference case [5].

Using the same notations as the previous section, $\mathbf{B}$ is now equal to

$$
\mathbf{B}=\left[\begin{array}{ccc}
z^{2}+\left(\zeta_{h}+\varepsilon \zeta+\beta \tilde{U}\right) z+\Omega^{2}+\varepsilon \gamma & x_{\alpha} z^{2}-\varepsilon \zeta \lambda z+\beta \tilde{U}^{2}-\varepsilon \gamma \lambda & -\varepsilon \zeta z-\varepsilon \gamma \\
x_{\alpha} z^{2}-(\nu \tilde{U}-\varepsilon \zeta \lambda) z-\varepsilon \gamma \lambda & r_{\alpha}^{2} z^{2}+\left(\zeta_{\alpha}+\varepsilon \zeta \lambda^{2}\right) z+r_{\alpha}^{2}-\nu \tilde{U}^{2}+\varepsilon \gamma \lambda^{2} & \varepsilon \zeta \lambda z+\varepsilon \gamma \lambda \\
-\zeta z-\gamma & \zeta \lambda z+\gamma \lambda & z^{2}+\zeta z+\gamma
\end{array}\right]
$$

The determinant of the matrix $\mathbf{B}$ takes the form of a $6^{\text {th }}$ order polynomial,

$$
\operatorname{det}(\mathbf{B})=a_{6} z^{6}+a_{5} z^{5}+a_{4} z^{4}+a_{3} z^{3}+a_{2} z^{2}+a_{1} z+a_{0},
$$

where $a_{1}$ to $a_{6}$ are detailed in Appendix A. The Routh-Hurwitz criterion is used to determine when one of the roots of $\operatorname{det}(\mathbf{B})$ has a positive real part.

This study leads to four independent conditions called $b_{5}, c_{5}, d_{5}$ and $e_{5}$, which read

$$
b_{5}=\left(a_{5} a_{4}-a_{6} a_{3}\right) / a_{5}>0, c_{5}=b_{5} a_{3}-a_{5} b_{4}>0, d_{5}=c_{5} b_{4}-c_{4} b_{5}>0 \text { and } e_{5}=d_{5} c_{4}-d_{4} c_{5}>0,
$$

with $b_{4}=\left(a_{5} a_{2}-a_{6} a_{1}\right) / a_{5}, b_{3}=a_{0}, c_{4}=\left(b_{5} a_{3}-b_{3} a_{5}\right) / b_{5}$ and $d_{4}=b_{3}$.

These four conditions are plotted as an example in Fig. 2 for $\zeta=0.15$. Each line corresponds to the points where one of the four expressions $b_{5}$ to $e_{5}$ becomes positive, as a function of the reduced eigenfrequency $\gamma$, and for increasing values of $\tilde{U}$. Thus, the flutter velocity corresponds to the lowest curve and the stability zone to the area below it, represented as a gray-shaded area in Fig. 2.

This calculation is then conducted for different values of $\zeta$ in order to find the optimal couple $\left(\gamma_{\text {opt }}, \zeta_{\text {opt }}\right)$ for which the flutter velocity is maximal. The result is shown in Fig. 3. The maximal reduced flutter velocity, obtained for $\gamma=0.462$ and $\zeta=0.11$, is equal to 1.255 , which means a 


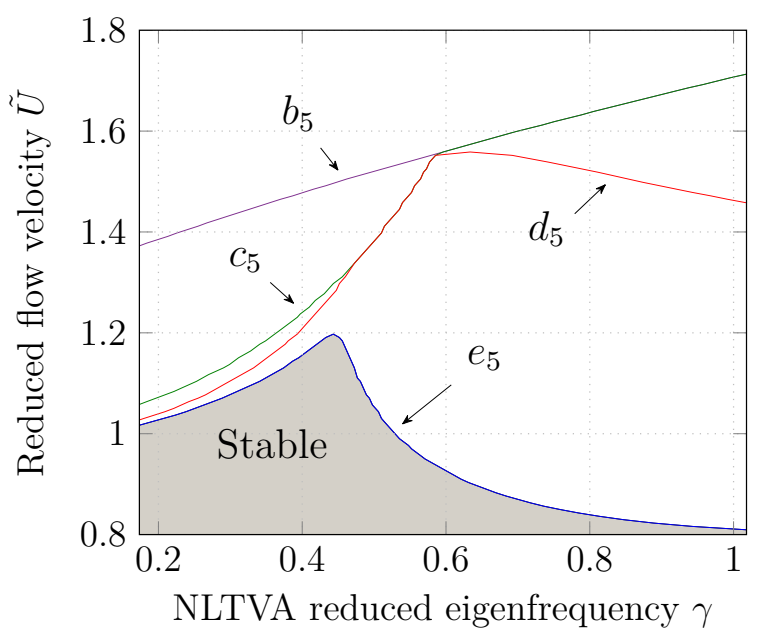

Figure 2: Reduced flutter velocity given by the Routh Hurwitz criterion as a function of the NLTVA reduced eigenfrequency $\gamma$, and for a damping ratio $\zeta=0.15$. The gray area corresponds to the stability of the system.

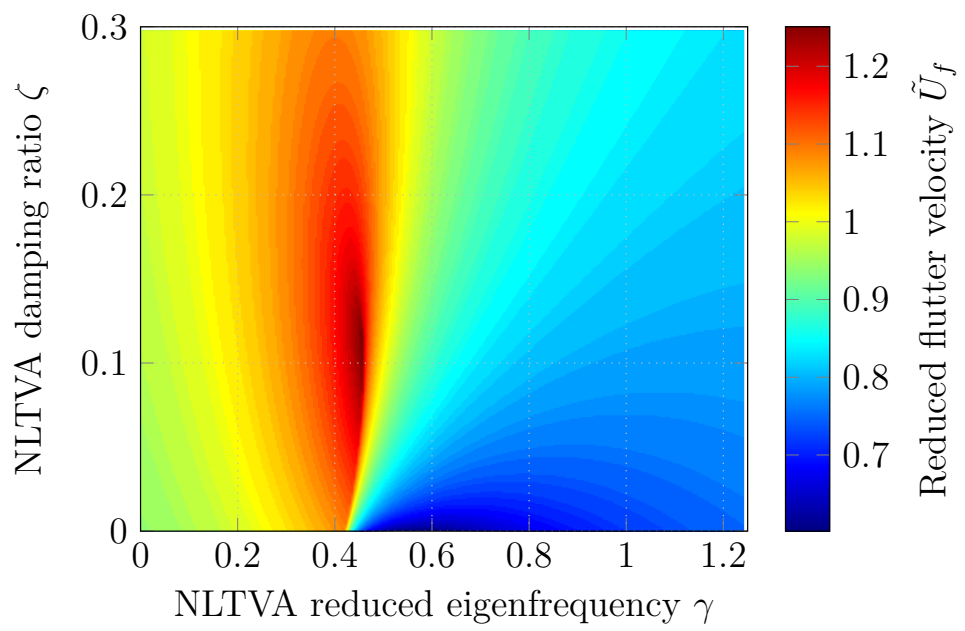

Figure 3: Cartography of the reduced flutter velocity given by the criterion $e_{5}$ as a function of the NLTVA reduced frequency $\gamma$ and damping ratio $\zeta$.

$34.5 \%$ gain as compared to the flutter velocity without absorber, which is equal to 0.934 . This gain on the flutter velocity is named $G_{\tilde{U}_{f}}$. Because of their complexity, the equations $b_{5}=0$, $c_{5}=0, d_{5}=0$ and $e_{5}=0$ cannot be easily made explicit with respect to $\zeta$ and $\gamma$. Thus, analytical expressions of the optimal values of $\zeta$ and $\gamma$ are not defined. Nevertheless these optimal values can be found numerically.

In practical situations, a slight detuning of $\gamma$ and $\zeta$ may occur. Thus, the sensitivity of $\tilde{U}_{f}$ regarding these parameters is detailed. It is shown in Fig. 3 that $\gamma$ has more influence on $\tilde{U}_{f}$ than 
$\zeta$. Moreover, $\tilde{U}_{f}^{\max }$ is on a sharp point rather than on a plateau. That is, if $\gamma$ increases a little, $\tilde{U}_{f}$ decreases significantly. Nevertheless, if $\gamma$ decreases a little, the $\tilde{U}_{f}$ 's drop is less abrupt. Therefore, the control should guarantee $\gamma \leq \gamma_{\text {opt }}$. For a more quantitative point of view, a $10 \%$ increase of $\gamma$ gives a $20 \%$ decrease of $\tilde{U}_{f}$ and a $10 \%$ decrease of $\gamma$ implies a $7 \%$ decrease of $\tilde{U}_{f}$, whereas a $10 \%$ increase or decrease of $\zeta$ gives a $4 \%$ decrease of $\tilde{U}_{f}$.

\subsection{Influence of the airfoil parameters}

In this section, the airfoil parameters influence on the NLTVA efficiency is investigated. Three main parameters are identified for characterizing the airfoil : the nondimensional static moment $x_{\alpha}=S_{\alpha} /(M b)$, which describes the coupling between pitch and plunge motion, the radius of

gyration $r_{\alpha}=\sqrt{I_{\alpha} /\left(M b^{2}\right)}$, which expresses the ratio between the airfoil inertias and finally the frequency ratio $\Omega=\omega_{h} / \omega_{\alpha}$ between the two natural frequencies of the plunge and pitch motion. Let us now define the investigation range for each of these three selected parameters.

The location of EC from the leading edge is equal to $0.45 c$ in the reference case. The selected investigation range is selected as [0 0.6]. The extreme value for $x_{\alpha}$ is $x_{\alpha}=1.1$; corresponding to a gravity center at the trailing edge, however this situation never occurs in practice. The value for $r_{\alpha}^{2}$ depends on the airfoil density distribution and shape. Although theoretically not bounded, it is generally in the interval $[01][26,30,31]$; which is thus the selected range in this study. Finally, variations of $\Omega^{2}$ are selected in the range $\left[\begin{array}{ll}0 & 1\end{array}\right]$. Indeed, the natural frequency of the pitch motion is generally larger than the plunge one, thus $\Omega^{2} \leq 1$.

The optimal value $\gamma_{\text {opt }}$ and $\zeta_{\text {opt }}$ are computed using the methodology described in section 3.1 while varying the three parameters $x_{\alpha}, r_{\alpha}^{2}$ and $\Omega^{2}$ in their respective ranges. The corresponding gain in flutter velocity, $G_{\tilde{U}_{f}}$, is plotted in Fig. 4 . The color represents the value of $G_{\tilde{U}_{f}}$ for each set of airfoil parameters. The influence of the NLTVA on $G_{\tilde{U}_{f}}$ is highly dependent on the airfoil parameters and can vary from $10 \%$ up to more than $140 \%$.

General tendencies on the influence of $x_{\alpha}, r_{\alpha}^{2}$ and $\Omega^{2}$ on the flutter velocity gain $G_{\tilde{U}_{f}}$ can be drawn from Fig. 4. First of all, there is a small strip of large $G_{\tilde{U}_{f}}$ for small values of $r_{\alpha}^{2}$, especially visible for $\Omega^{2}=0.4$ and 0.7. This small strip has a singular behavior, but the corresponding value of $r_{\alpha}^{2}$ are particularly small and not very realistic. Otherwise, the influence of $r_{\alpha}^{2}$ is limited. Regarding $\Omega^{2}$, the larger it is the larger is $G_{\tilde{U}_{f}}$. Indeed, when $\Omega^{2}$ is close to one, $\omega_{\alpha}$ and $\omega_{h}$ are close to each other. In this case, the efficiency of the NLTVA, working optimally at a single frequency, is enhanced since both degrees of freedom can be controlled. Eventually, the influence of $x_{\alpha}$ is substantial, the NLTVA effectiveness decreasing while $x_{\alpha}$ is increasing. The explanation of this tendency is not obvious. An argument that can be given is that a large $x_{\alpha}$ push the two airfoil coupled frequencies apart. Thus, as for $\Omega^{2}$, the same causes producing the same effects, if $x_{\alpha}$ is large the NLTVA is less efficient.

\subsection{NLTVA linear parameters tuning rule}

The aim of the present section is to provide a simple tuning rule allowing one to compute easily the optimal NLTVA linear parameters, namely $\gamma_{\text {opt }}$ and $\zeta_{\text {opt }}$; as a function of the airfoil parameters, condensed in the three dimensionless parameters $x_{\alpha}, r_{\alpha}^{2}$ and $\Omega^{2}$. For that purpose, a third order 


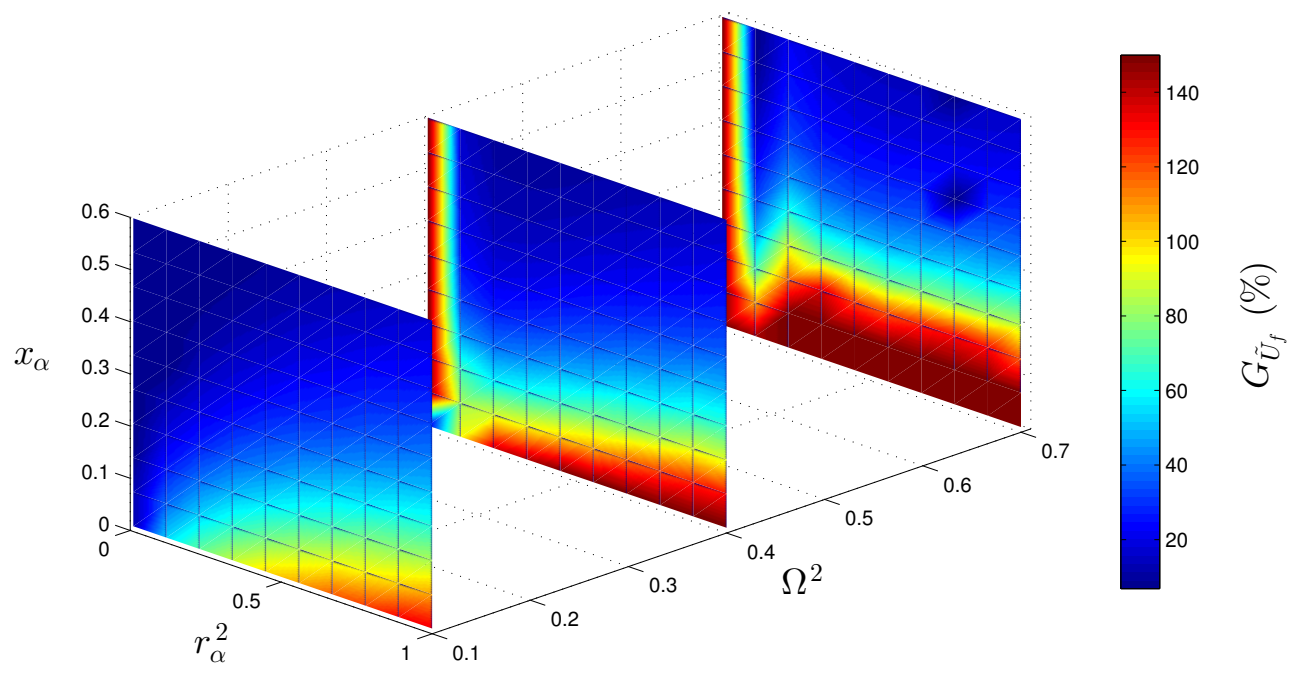

Figure 4: Evolution of the optimal flutter velocity gain $G_{\tilde{U}_{f}}$ caused by the NLTVA vs $x_{\alpha}, r_{\alpha}^{2}$ and $\Omega^{2}$.

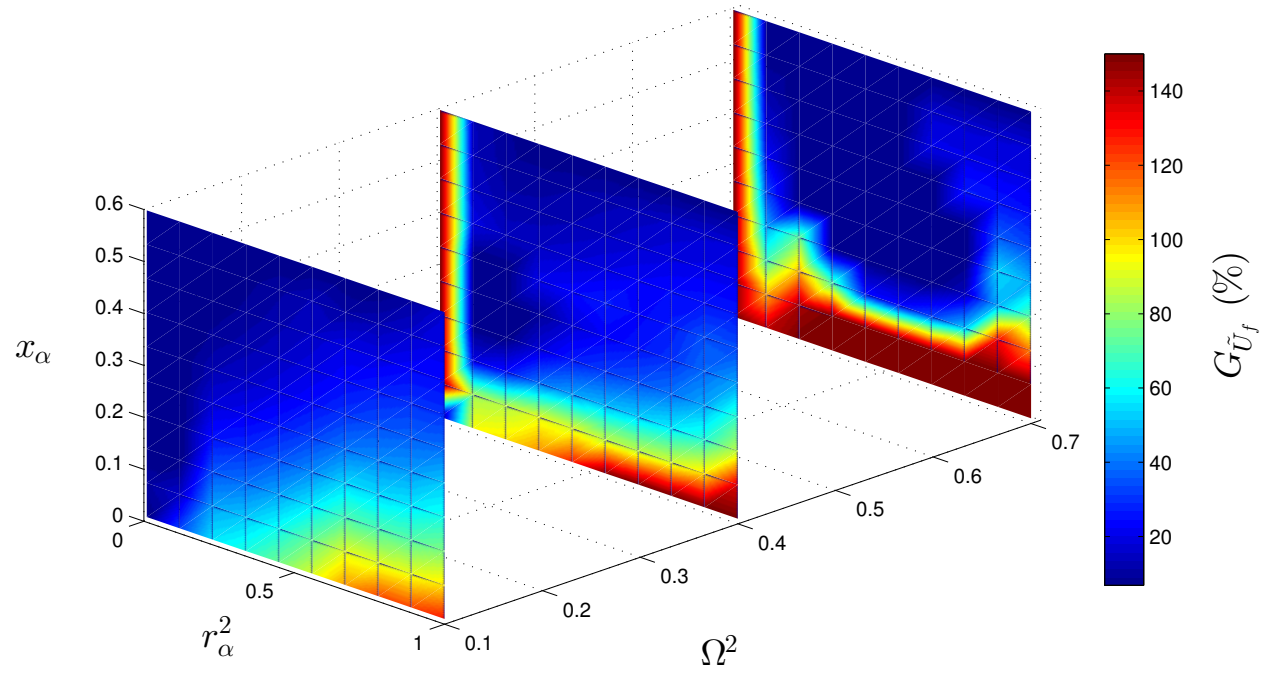

Figure 5: Estimation of $G_{\tilde{U}_{f}}$ using the fitted parameters $\zeta_{\mathrm{opt}}^{\mathrm{fit}}$ and $\gamma_{\mathrm{opt}}^{\mathrm{fit}}$ vs $x_{\alpha}, r_{\alpha}^{2}$ and $\Omega^{2}$.

polynomial expansion has been found to give an accurate fit to the hypersurface shown in Fig. 4 . 
More specifically, the following expansion has been used

$$
\begin{aligned}
& \gamma_{\mathrm{opt}}^{\mathrm{fit}}=\sum_{i, j, k=0}^{3} a_{i j k} x_{\alpha}^{i}\left(r_{\alpha}^{2}\right)^{j}\left(\Omega^{2}\right)^{k} \\
& \zeta_{\mathrm{opt}}^{\mathrm{fit}}=\sum_{i, j, k=0}^{3} b_{i j k} x_{\alpha}^{i}\left(r_{\alpha}^{2}\right)^{j}\left(\Omega^{2}\right)^{k}
\end{aligned}
$$

the coefficients of which are given in Appendix B. The resulting $G_{\tilde{U}_{f}}$, calculated with $\gamma_{\text {opt }}^{\text {fit }}$ and $\zeta_{\text {opt }}^{\text {fit }}$, is plotted in Fig. 5. This figure has to be compared with Fig. 4, showing that the qualitative and quantitative features are retrieved. Hence, Eq. (6) can be easily used in order to determine directly the NLTVA linear parameters as a function of its airfoil parameters.

\section{Nonlinear analysis of the post-critical regime}

This section is devoted to the tuning methodology for optimizing the nonlinear stiffness of the NLTVA. Two goals are in view. First, one would like to ensure a supercritical bifurcation, as being a safer scenario for an engineering design with respect to the global stability of the airfoil. Second, the post-critical LCOs are investigated in order to decrease their amplitudes as much as possible. For the nonlinear analysis, the NLTVA linear parameters correspond to the airfoil reference case and are gathered in Table 2.

\begin{tabular}{cccc}
\hline$\varepsilon$ & $\lambda$ & $\zeta$ & $\gamma$ \\
\hline 0.05 & 1 & 0.11 & 0.462 \\
\hline
\end{tabular}

Table 2: NLTVA linear parameters used for the nonlinear analysis.

\subsection{Criticality analysis}

The Hopf bifurcation encountered at $\tilde{U}=\tilde{U}_{f}$ can be either subcritical or supercritical. In a normal form analysis of the bifurcation, this characteristic is simply related to the sign of the cubic nonlinear term when the system is written in polar form $[32,33,34]$. The aim of this section is to obtain the expression of this coefficient, based on a reduced-order model that contains the leading order nonlinearity of the normal form. The technique used follows classical methods for dynamical systems $[32,35]$ that have been recently applied on a Van der Pol-Duffing oscillator [20]. It is decomposed into five steps.

The first step is to recast the equations of motion (2) as a first-order dynamical system as follows

$$
\tilde{\mathbf{q}}^{\prime}=\mathbf{A} \tilde{\mathbf{q}}+\mathbf{b}
$$


$\tilde{\mathbf{q}}=\left[\begin{array}{c}y \\ \alpha \\ \tilde{x} \\ y^{\prime} \\ \alpha^{\prime} \\ \tilde{x}^{\prime}\end{array}\right], \mathbf{A}=\left[\begin{array}{cc}0_{3} & I d_{3} \\ -\mathbf{M}^{-1} \mathbf{K} & -\mathbf{M}^{-1} \mathbf{C}\end{array}\right], \mathbf{b}=\left[\begin{array}{c}0 \\ 0 \\ 0 \\ -\mathbf{M}^{-1}\left[\begin{array}{c}\xi_{h} y^{3}+\varepsilon \xi(y-\tilde{x}-\lambda \alpha)^{3} \\ \xi_{\alpha} \alpha^{3}+\varepsilon \xi \lambda(\tilde{x}-y+\lambda \alpha)^{3} \\ \xi(\tilde{x}-y+\lambda \alpha)^{3}\end{array}\right]\end{array}\right]$.

For the criticality analysis, $\xi_{\alpha}, \xi_{h}$ and $\xi$ are left variable, $\xi$ being the nonlinear control parameter of the NLTVA.

The second step is to estimate the eigenvalues and eigenvectors of $\mathbf{A}$ for $\tilde{U}=\tilde{U}_{f}$. Because the expression of $\mathbf{A}$ is particularly tedious, an analytical expression of its eigenvectors and eigenvalues is not reachable, they are thus calculated using the airfoil parameters from Table 1 and the retained NLTVA linear parameters. The system being of dimension 6 , the eigenvalues and eigenvectors of A for $\tilde{U}=\tilde{U}_{f}$ are denoted respectively $d_{1}$ to $d_{6}$ and $\mathbf{V}_{1}$ to $\mathbf{V}_{6}$. Besides, the real and imaginary part of $d_{1 . .6}$ are denoted $\lambda_{1 . .6}$ and $\omega_{1 . .6}$ respectively. The Hopf bifurcation is characterized by a pair of complex conjugate eigenvalues crossing the imaginary axis. At criticality for $\tilde{U}=\tilde{U}_{f}$, these eigenvalues, denoted $d_{1}$ and $d_{2}$, have a zero real part and read

$$
d_{1}=i \omega_{1} \quad \text { and } \quad d_{2}=i \omega_{2}=-i \omega_{1}
$$

Their corresponding eigenvectors are denoted $\mathbf{V}_{1}$ and $\mathbf{V}_{2}$. Because it has been found that in our case $\mathbf{V}_{1}$ to $\mathbf{V}_{6}$ are complex conjugate, the following transformation matrix is introduced [32, 20],

$$
\mathbf{T}=\left[\operatorname{Re}\left(\mathbf{V}_{1}\right) \operatorname{Im}\left(\mathbf{V}_{1}\right) \operatorname{Re}\left(\mathbf{V}_{3}\right) \operatorname{Im}\left(\mathbf{V}_{3}\right) \operatorname{Re}\left(\mathbf{V}_{5}\right) \operatorname{Im}\left(\mathbf{V}_{5}\right)\right] .
$$

The third step is to obtain the first-order Jordan form of Eq. (7) by changing the linear basis with the transform $\tilde{\mathbf{q}}=\mathbf{T y}$. The dynamics for $\mathbf{y}$ thus writes

$$
\mathbf{y}^{\prime}=\mathbf{W} \mathbf{y}+\tilde{\mathbf{b}}
$$

where $\tilde{\mathbf{b}}=\mathbf{T}^{-1} \mathbf{b}$ and $\mathbf{W}=\mathbf{T}^{-1} \mathbf{A T}$. It is reminded to the reader that in this expression, the elements of $\tilde{\mathbf{b}}$ are function of $\xi_{\alpha}, \xi_{h}$ and $\xi$.

The fourth step is to reduce Eq. (8) to its first-order center manifold [32, 33, 36, 37]. For a system encompassing only third order nonlinearities, this corresponds to neglect variables not related to the bifurcation, i.e. $y_{3}$ to $y_{6}$.

$$
\left[\begin{array}{l}
y_{1}^{\prime} \\
y_{2}^{\prime}
\end{array}\right]=\left[\begin{array}{cc}
0 & \omega_{1} \\
-\omega_{1} & 0
\end{array}\right]\left[\begin{array}{l}
y_{1} \\
y_{2}
\end{array}\right]+\left[\begin{array}{l}
\tilde{b}_{1} \\
\tilde{b}_{2}
\end{array}\right],
$$

where $\tilde{b}_{1}$ and $\tilde{b}_{2}$ are the following polynomial functions

$$
\begin{aligned}
& \tilde{b}_{1}=c_{30}\left(\xi_{\alpha}, \xi_{h}, \xi\right) y_{1}^{3}+c_{21}\left(\xi_{\alpha}, \xi_{h}, \xi\right) y_{1}^{2} y_{2}+c_{12}\left(\xi_{\alpha}, \xi_{h}, \xi\right) y_{1} y_{2}^{2}+c_{03}\left(\xi_{\alpha}, \xi_{h}, \xi\right) y_{2}^{3} \\
& \tilde{b}_{2}=d_{30}\left(\xi_{\alpha}, \xi_{h}, \xi\right) y_{1}^{3}+d_{21}\left(\xi_{\alpha}, \xi_{h}, \xi\right) y_{1}^{2} y_{2}+d_{12}\left(\xi_{\alpha}, \xi_{h}, \xi\right) y_{1} y_{2}^{2}+d_{03}\left(\xi_{\alpha}, \xi_{h}, \xi\right) y_{2}^{3}
\end{aligned}
$$

The expression of the $c_{i j}\left(\xi_{\alpha}, \xi_{h}, \xi\right)$ and $d_{i j}\left(\xi_{\alpha}, \xi_{h}, \xi\right)$ coefficients are given in Appendix C. The fifth step is to operate the following polar transformation

$$
y_{1}=r \cos \left(\omega_{1}\right) \text { and } y_{2}=r \sin \left(\omega_{1}\right),
$$


where $r \in \mathbb{R}$. Unfolding the singularity in the vicinity of the critical point [34, 33], the Hopf bifurcation can be locally described with

$$
r^{\prime}=\lambda_{\alpha}\left(\tilde{U}-\tilde{U}_{f}\right) r+\rho r^{3},
$$

where $\lambda_{\alpha}=\partial \lambda / \partial \tilde{U}$ and $\lambda=\lambda_{1}$ for any $\tilde{U} ; \lambda_{\alpha}$ is estimated numerically. That is, $\lambda_{1}$ is calculated for a flow speed $\tilde{U}$ slightly larger than $\tilde{U}_{f}(\sim 0.05 \%)$ and the slope between the two is calculated. It has to be noticed that Eq. $(11)$ is only valid for $\tilde{U}$ in a neighborhood of $\tilde{U}_{f}$, because Eqs. $(9-11)$ result from a local analysis at criticality. The validity of this neighborhood will be discussed in the next section. Eventually, it can be proven [33] that

$$
\rho=(1 / 8)\left(3 c_{30}+c_{12}+d_{21}+3 d_{03}\right)
$$

The non-trivial solution of Eq. (11) is equal to $\sqrt{-\lambda_{\alpha}\left(\tilde{U}-\tilde{U}_{f}\right) / \rho}$. Thus, if $\rho>0$, this solution is valid for $\tilde{U}<\tilde{U}_{f}$ and the bifurcation is subcritical. Otherwise, if $\rho<0$, the non-trivial solution of Eq. (11) is valid for $\tilde{U}>\tilde{U}_{f}$ and the bifurcation is supercritical. The value of $\xi$ for which $\rho=0$ (denoted $\xi_{c}$ ) is a linear function of $\xi_{\alpha}$ and $\xi_{h}$, and can directly be derived from Eq. (12). The expression of $\xi_{c}$ is found by using the parameters from Table 1 and 2 and yields :

$$
\xi_{c}=0.0116 \xi_{h}+0.0966 \xi_{\alpha} .
$$

If $\xi>\xi_{c}$, the instability is supercritical and if $\xi<\xi_{c}$, the instability is subcritical. The nonlinearity of the NLTVA is thus able to cancel the subcriticality of the bifurcation. The validation of the analytical procedure described above as well as the behavior of the system in the post-critical regime is investigated in the next section.

\subsection{Post-critical regime}

The analytical procedure described above is compared with numerical solutions obtained by a continuation technique, using a pseudo-arclength method implemented in the software AUTO [38].

The post-critical regime is explored for a hard and a soft pitch spring, i.e. $\xi_{\alpha}>0$ and $\xi_{\alpha}<0$ respectively. A hard spring can be used to represent a thin wing or a propeller blade, whereas a soft spring may be associated with panel buckling [39, 24]. Nonlinear restoring force is considered only on the pitch motions so that $\xi_{h}=0$. This assumption is justified by the fact that in the post-critical regime, LCOs amplitudes are very small on the plunge mode, so that the cubic nonlinearity is not excited. Moreover, it has been verified in all the numerical results presented that setting $\xi_{h}=1$ does not change significantly the bifurcation behavior.

Firstly, the hard pitch spring is investigated. This configuration is similar to the one studied by Lee et al. [24]. Secondly, the configuration with a soft pitch spring is explored. This case is similar to the one studied by Pettit and Beran [25]. In this case a quintic stiffness is added to the pitch stiffness in order to have stable solutions in the post-critical regime. 


\subsubsection{Hard pitch spring}

In a similar manner as in [24], we set here the nonlinear restoring forces to $\xi_{\alpha}=1$ and $\xi_{h}=0$. The bifurcation diagram for the airfoil without absorber (i.e. with $\varepsilon=0$ ) is first shown in Fig. 6(a,b). The result corresponds to a supercritical Hopf bifurcation. The analytical solution obtained in section 4.1 is also represented, showing a very good agreement for small amplitude oscillations.
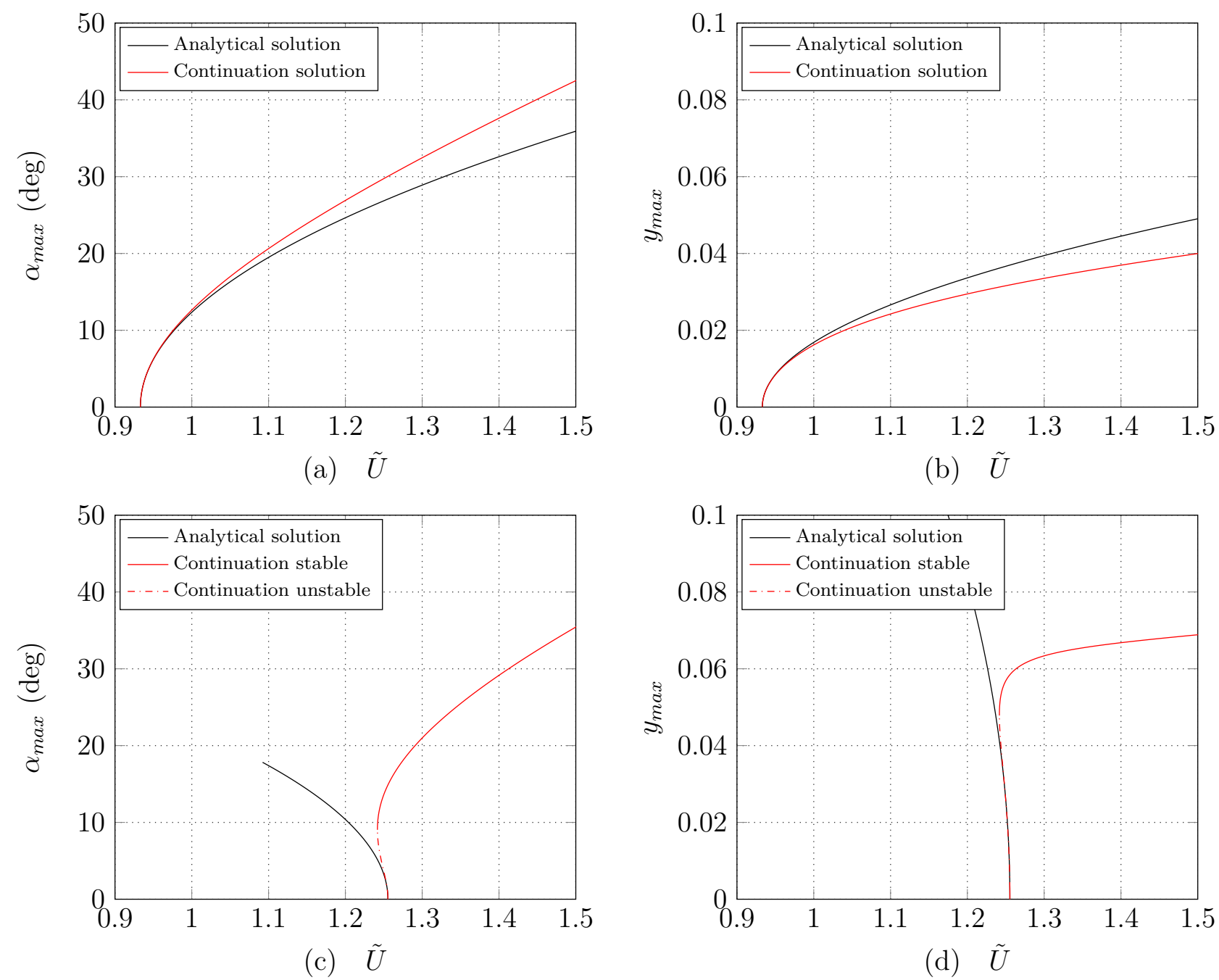

Figure 6: Bifurcation diagram of pitch and plunge mode for the system without absorber $(a, b)$ and in presence of a NLTVA without nonlinearities $(\mathrm{c}, \mathrm{d})$.

The case with a NLTVA without nonlinearities (i.e. $\xi=0$ ), which is equivalent to a TMD, is shown in Fig. 6(c,d). As predicted by the linear stability analysis, the flutter velocity is repelled from 0.93 to 1.26. Nevertheless, the Hopf bifurcation becomes subcritical. Hence, a detrimental effect of repelling the flutter velocity is to shift the originally supercritical Hopf bifurcation to a 
subcritical one. The most salient disadvantage of subcriticality lies in the appearance of a jump phenomenon at the flutter velocity, when increasing (and decreasing) the flow speed. In this example, when $\tilde{U}$ is slightly larger than $\tilde{U}_{f}$, the amplitude of pitch motion varies suddenly from 0 to 15 degrees, which may cause serious damage to the structure. Another effect of the subcriticality is the existence of a bi-stable region for flow velocities smaller than the flutter velocity.

We now investigate the influence of the nonlinear stiffness $\xi$ of the NLTVA on the Hopf bifurcation criticality. For that purpose, the bifurcation diagram is plotted for four increasing values of $\xi$ in Fig. 7. The four selected cases are

1. $\xi=0.06\left(\xi<\xi_{c}\right)$, where the subcriticality is less pronounced than for $\xi=0$,

2. $\xi=0.0966\left(\xi=\xi_{c}\right)$, where, according to the analytical prediction, there is a transition between subcritical and supercritical behavior,

3. $\xi=0.217\left(\xi_{c}<\xi<\xi_{s}\right.$, where $\left.\xi_{s}=0.218\right)$, which is the largest value of $\xi$ before a global bifurcation appearence,

4. $\xi=0.218\left(\xi=\xi_{s}\right)$, where a global bifurcation suddenly occurs in the diagram, with appearance of two limit points, as well as two Neimarck-Sacker bifurcations points with in between quasiperiodic solutions.

These results clearly evidenced the effect of the NLTVA on the bifurcation diagram. Increasing the cubic stiffness $\xi$ turns out to make the bifurcation more and more supercritical. However the global behavior is also affected so that the optimal value is not the largest possible value of $\xi$. The global bifurcation occurring for $\xi_{s}=0.218$ modifies drastically the diagram, which is then non optimal in a design point of view. Indeed, the two limit points give rise to a new global subcritical behavior with the appearance of a solution branch with large amplitude that persists for smaller values of $\tilde{U}_{f}$. Hence, this situation is to be avoided and optimal values for $\xi$ are founded with $\xi>\xi_{c}$ (ensuring supercriticality) and $\xi<\xi_{s}$ (avoiding an extra global bifurcation scenario); the largest decrease of LCO amplitude on pitch being obtained with $\xi=0.217$. This case could be optimal, but its robustness is not satisfactory since $\xi=0.218$ gives a detrimental global bifurcation scenario. Furthermore, the abrupt and drastic transition of the scenario for $\xi$ varying from $\xi=0.217$ to $\xi=0.218$ suggests that periodic stable solutions probably exist also for $\xi<0.218$ and $\tilde{U}<\tilde{U}_{f}$. However, a detailed analysis of this aspect is out of the scope of this study.

The influence of the NLTVA is summarized in Fig. 8. The LCO amplitude on pitch has been reduced significantly by the NLTVA. For example at $\tilde{U}=1.4$, the LCO amplitude on pitch has been decreased by $36.4 \%$ compared to the case without absorber. Nevertheless, at the same time, the LCO amplitude on plunge has been increased by $115.9 \%$. Even if this increase seems large, it is remarked that the more inconvenient LCO are those on the pitch mode, because this is the most energetic mode of the system in the post-critical regime and even after an increase of $115.9 \%$ the LCO amplitude on plunge remains very small (8\% of the semi-chord). 

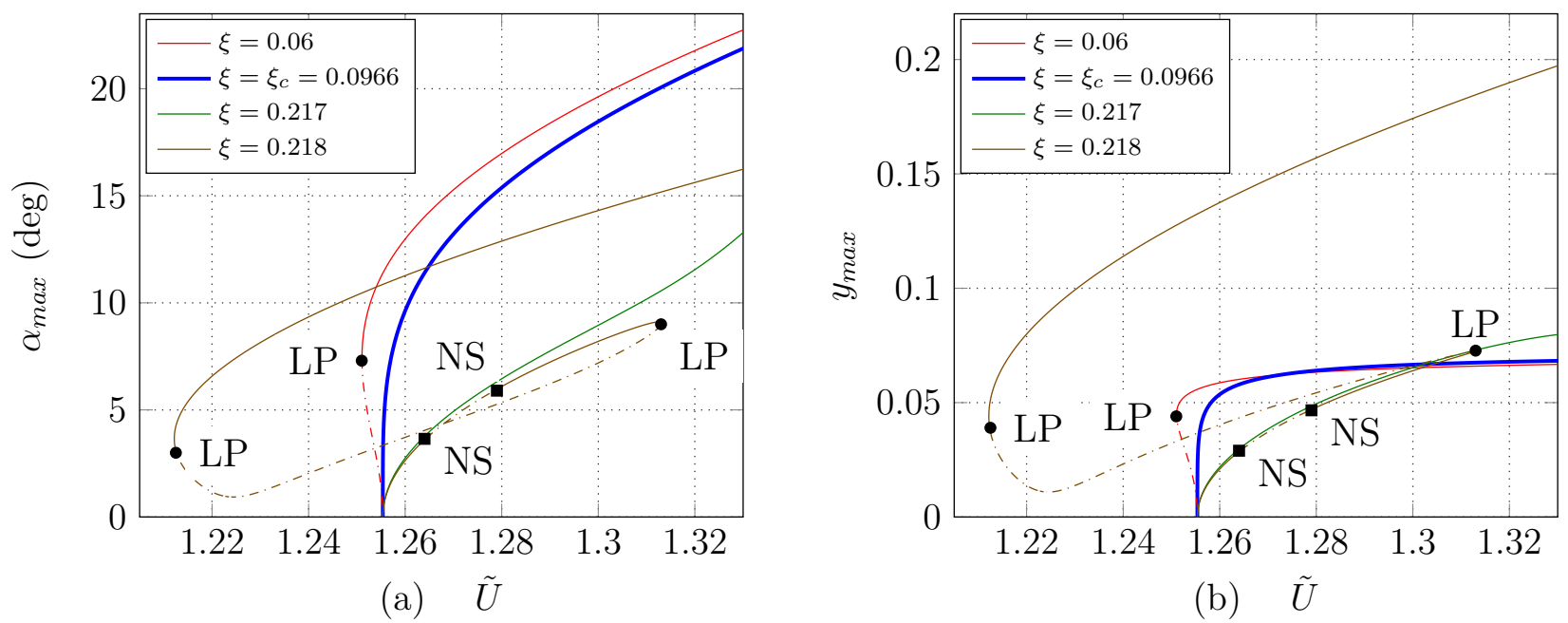

Figure 7: Bifurcation diagram of (a) pitch and (b) plunge mode for four increasing values of $\xi$. The solid lines correspond to the stable solutions and the dashed line to the unstable ones. LP stands for limit point and NS for Neimark Sacker bifurcation point.

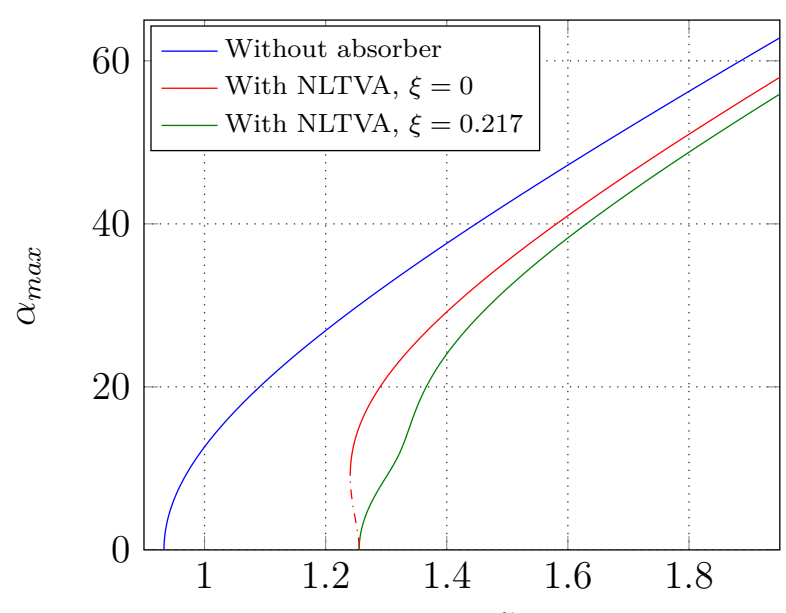

(a) $\tilde{U}$

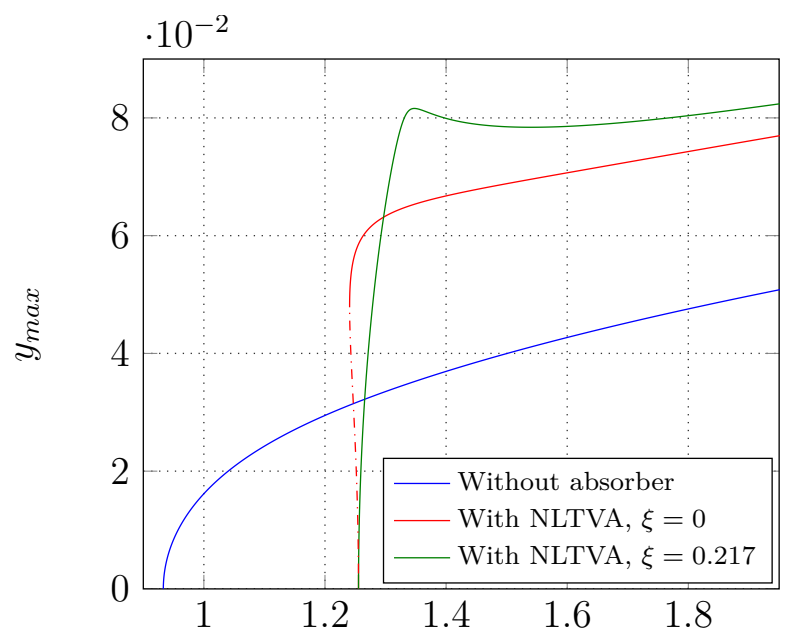

(b) $\tilde{U}$

Figure 8: Influence of the NLTVA with $\xi=0$ and $\xi=0.217$ on the bifurcation diagram of the 2 dofs airfoil. 


\subsubsection{Soft pitch spring}

In this section, the influence of the NLTVA on the airfoil with a soft pitch spring is investigated. That is $\xi_{\alpha}=-1$ and $\xi_{h}=0$. Moreover, a quintic stiffness, named $\xi_{\alpha 5}$, is added to the pitch restoring force, which means that the vector $\mathbf{b}$ from Eq. (7) becomes

$$
\mathbf{b}=\left[\begin{array}{c}
0 \\
0 \\
0 \\
-\mathbf{M}^{-1}\left[\begin{array}{c}
\xi_{h} y^{3}+\varepsilon \xi(y-\tilde{x}-\lambda \alpha)^{3} \\
\xi_{\alpha} \alpha^{3}+\xi_{\alpha 5} \alpha^{5}+\varepsilon \xi \lambda(\tilde{x}-y+\lambda \alpha)^{3} \\
\xi(\tilde{x}-y+\lambda \alpha)^{3}
\end{array}\right]
\end{array}\right]
$$

We choose in this study $\xi_{\alpha 5}=7$, this configuration is similar to the case studied by Pettit and Beran [25].

As in the previous section, the effect of $\xi$ on the airfoil bifurcation diagram is investigated. For that purpose, the bifurcation diagram is computed for five increasing values of $\xi$ from -0.5 to 0.4 , the results are shown in Fig. 9. The key values are the following:

- $\xi_{c}=-0.0966$, which is the critical value predicted from the analytical study using Eq. (13). When $\xi \geq \xi_{c}$, the bifurcation is supercritical, otherwise it is subcritical.

- $\xi_{s_{1}}=-0.15$ and $\xi_{s_{2}}=0.125$ : between these two values, a global bifurcation occurs and the solution branches encounters Neimarck-Sacker bifurcations, resulting in a more complex shape of the diagram.

- $\xi=0$ which corresponds to the TMD.

Around the criticality, detailed in Fig. 9(c,d), the effect of increasing $\xi$ is to bend the bifurcation branch to the right. In order to have a supercritical behavior, one would have thus expect that selecting a larger value than $\xi_{c}=-0.0966$ could have been sufficient. However the numerical simulation shows that ensuring supercriticality with $\xi \geq \xi_{c}$ in the vicinity of the critical flutter velocity do not avoid the occurrence of a global subcritical behavior. Increasing $\xi$ worsens the scenario with a large amplitude branch going down to smaller velocities, even though locally the supercriticality is enforced. In particular the cases $\xi=\xi_{c}=-0.0966$ and $\xi=0$ shown in Fig. 9(a,b) show large amplitudes especially present in the plunge mode, which are not acceptable in a design perspective to mitigate the flutter instability. And still increasing $\xi$ is definitely not a solution, as the situation worsens as soon as $\xi>\xi_{s_{2}}$, as exemplified with the case $\xi=0.4$.

In this more difficult case, a compromise must be found between the acceptable subcriticality and the amplitudes of LCOs in the post-critical regime. With this aim in view, the case $\xi=-0.5$ shown in Fig. 9 could represent such an optimal, with a small interval of subcriticality together with reduced amplitude of the LCOs. Depending on the context and the design constraints, $\xi=-0.15$ also represents an interesting case for the control of the flutter instability. Nevertheless this case is more difficult to handle than the precedent with a hardening spring, and an optimal control as initially desired is out of reach with the present NLTVA design. 


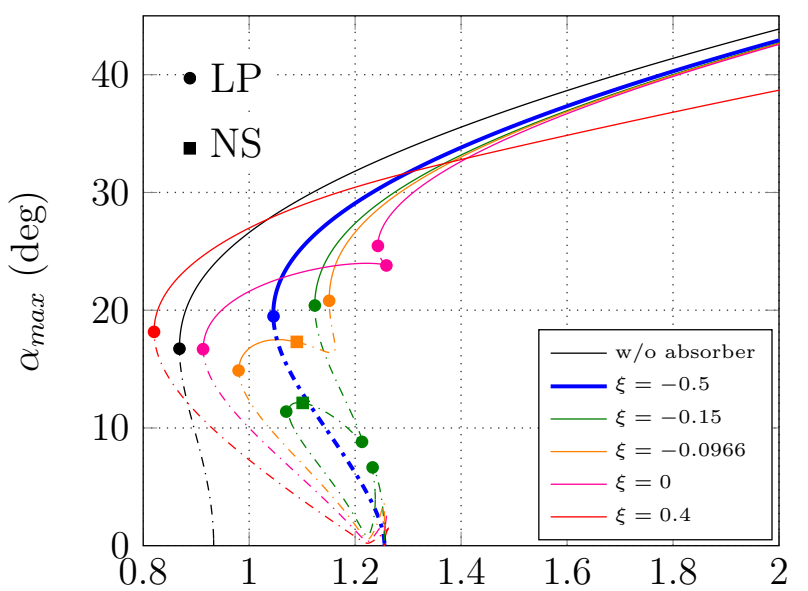

(a) $\tilde{U}$

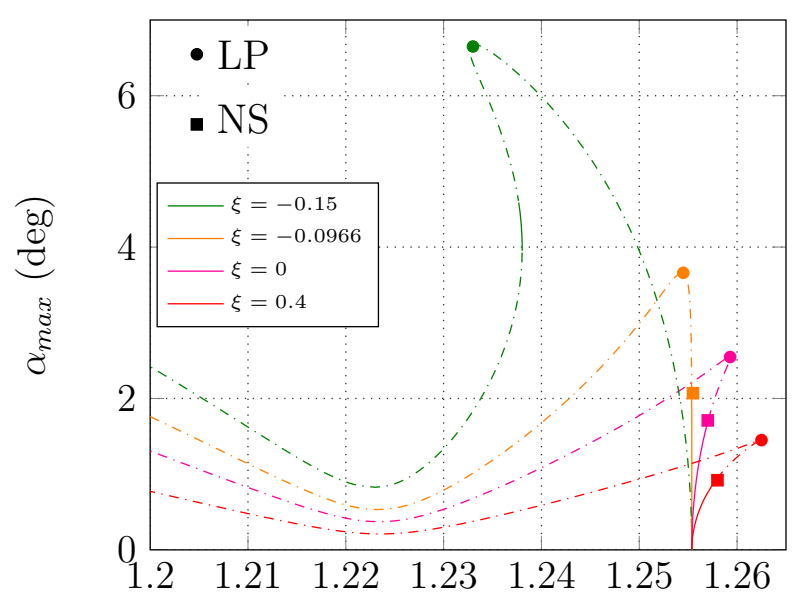

(c) $\tilde{U}$

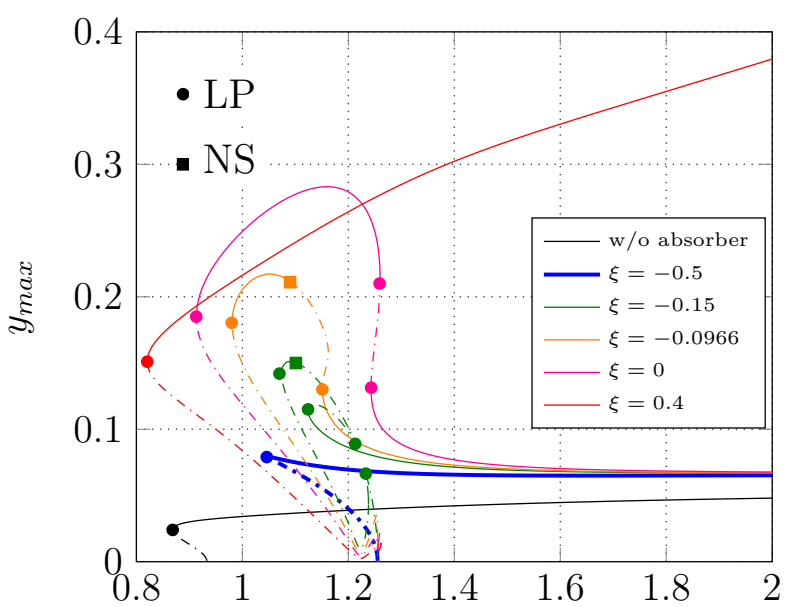

(b) $\tilde{U}$

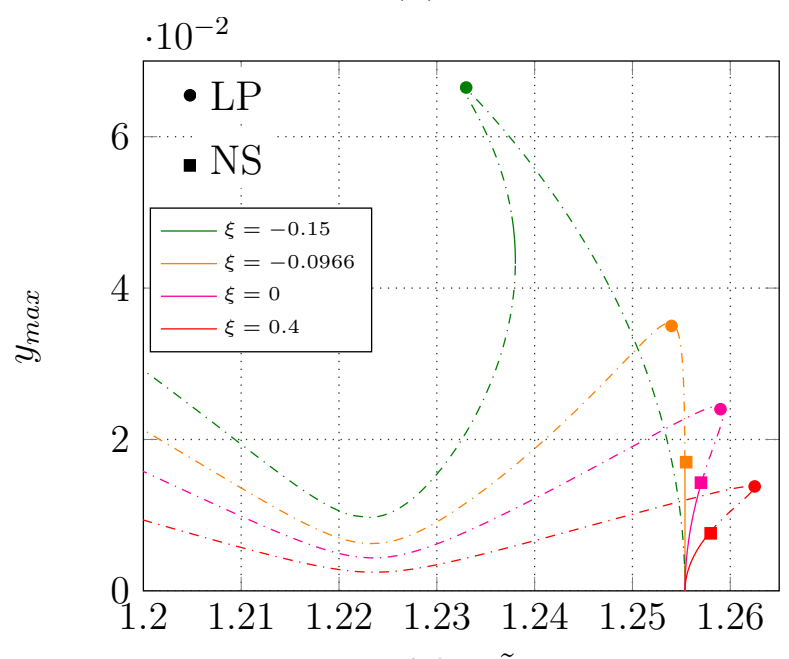

(d) $\tilde{U}$

Figure 9: Bifurcation diagram of (a,c) pitch and (b,d) plunge mode for increasing values of $\xi$. The solid lines correspond to the stable solutions and the dashed line to the unstable ones. The plots (c) and (d) detail the bifurcation diagram around the criticality for $\xi \geq-0.15$. LP stands for limit point and NS for Neimark Sacker bifurcation point. 


\section{Conclusion}

The influence of an NLTVA on the airfoil flutter instability has been investigated. The NLTVA is defined by two parameters : its linear stiffness, which can be tuned in order to repel as much as possible the flutter velocity $U_{f}$; and its nonlinear stiffness, which can be used for a better control of the bifurcation diagram. Linear stability analysis has shown that the flutter velocity can indeed be repelled by finding optimal values of stiffness together with the damping ratio of the absorber. Important gains ranging roughly from $30 \%$ to more than $150 \%$ can be theoretically achieved. A design rule has also been provided by means of a polynomial fit of the optimal parameters, for varying values defining the studied airfoil.

A nonlinear analysis has then be conducted, including a local analytical prediction, and a bifurcation study for a hardening and a softening pitch spring stiffness. Results show that in the hardening case, an optimal tuning is achieved, controlling the bifurcation in a supercritical case with an important gain in the LCOs amplitudes. The softening case has been found more difficult to optimize, and a compromise between subcriticality and large amplitude LCOs has to be found, depending on the context.

\section{Acknowledgment}

We thank Direction Générale de l'Armement (DGA) for a financial support to this work.

\section{References}

[1] Vipperman, J. S., Clark, R. L., Conner, M., and Dowell, E. H., 1998. "Experimental active control of a typical section using a trailing-edge flap". Journal of Aircraft, 35(2), pp. 224-229.

[2] Karpel, M., 1982. "Design for active flutter suppression and gust alleviation using state-space aeroelastic modeling". Journal of Aircraft, 19(3), pp. 221-227.

[3] Ko, J., Kurdila, A. J., and Strganac, T. W., 1997. "Nonlinear control of a prototypical wing section with torsional nonlinearity". Journal of Guidance, Control, and Dynamics, 20(6), pp. 1181-1189.

[4] Heeg, J., 1993. Analytical and experimental investigation of flutter suppression by piezoelectric actuation. Tech. Rep. 3241, NASA.

[5] Dowell, E. H., 2004. A Modern Course in Aeroelasticity. Kluwer Academic Publishers.

[6] Kwon, S.-D., and Park, K.-S., 2004. "Suppression of bridge flutter using tuned mass dampers based on robust performance design". Journal of Wind Engineering and Industrial Aerodynamics, $\mathbf{9 2 ( 1 1 ) , ~ p p . ~ 9 1 9 - 9 3 4 . ~}$

[7] Vaurigaud, B., Manevitch, L. I., and Lamarque, C.-H., 2011. "Passive control of aeroelastic instability in a long span bridge model prone to coupled flutter using targeted energy transfer". Journal of Sound and Vibration, 330(11), pp. 2580-2595. 
[8] Pourzeynali, S., and Datta, T., 2002. "Control of flutter of suspension bridge deck using TMD". Wind and Structures, 5(5), pp. 407-422.

[9] Den Hartog, J. P., 1934. Mechanical vibrations. McGraw-Hill Book Company, Inc., New York.

[10] Gattulli, V., Di Fabio, F., and Luongo, A., 2004. "Nonlinear tuned mass damper for selfexcited oscillations". Wind and Structures, 7, pp. 251-264.

[11] Lee, Y. S., Vakakis, A., Bergman, L., McFarland, D. M., and Kerschen, G., 2007. "Suppressing aeroelastic instability using broadband passive targeted energy transfers, part 1: theory". AIAA journal, 45(3), pp. 693-711.

[12] Lee, Y. S., Kerschen, G., McFarland, D. M., Hill, W. J., Nichkawde, C., Strganac, T. W., Bergman, L. A., and Vakakis, A. F., 2007. "Suppressing aeroelastic instability using broadband passive targeted energy transfers, part 2: experiments". AIAA journal, 45(10), pp. 2391-2400.

[13] Gendelman, O. V., 2001. "Transition of energy to a nonlinear localized mode in a highly asymmetric system of two oscillators". Nonlinear dynamics, 25(1-3), pp. 237-253.

[14] Vakakis, A. F., and Gendelman, O., 2001. "Energy pumping in nonlinear mechanical oscillators: part ii - resonance capture". Journal of Applied Mechanics, 68(1), pp. 42-48.

[15] Vakakis, A. F., Manevitch, L. I., Gendelman, O., and Bergman, L., 2003. "Dynamics of linear discrete systems connected to local, essentially non-linear attachments". Journal of Sound and Vibration, 264(3), pp. 559-577.

[16] Vakakis, A. F., Gendelman, O. V., Bergman, L. A., McFarland, D. M., Kerschen, G., and Lee, Y. S., 2008. Nonlinear targeted energy transfer in mechanical and structural systems, Vol. 156. Springer, Ontario.

[17] Viguié, R., and Kerschen, G., 2009. "Nonlinear vibration absorber coupled to a nonlinear primary system: a tuning methodology". Journal of Sound and Vibration, 326(3), pp. 780793.

[18] Habib, G., Detroux, T., Viguié, R., and Kerschen, G., 2015. "Nonlinear generalization of Den Hartog's equal-peak method". Mechanical Systems and Signal Processing, 52, pp. 17-28.

[19] Habib, G., and Kerschen, G., 2016. "A principle of similarity for nonlinear vibration absorbers". Physica D: Nonlinear Phenomena, 332(1), pp. 1-8.

[20] Habib, G., and Kerschen, G., 2015. "Suppression of limit cycle oscillations using the nonlinear tuned vibration absorber". In Proceedings of the Royal Society of London, Vol. 471, p. 20140976. 
[21] Grappasonni, C., Habib, G., Detroux, T., and Kerschen, G., 2016. "Experimental demonstration of a 3D-printed nonlinear tuned vibration absorber". In Nonlinear Dynamics, Volume 1. Conference Proceedings of the Society for Experimental Mechanics Series. Springer, pp. 173183.

[22] Benacchio, S., Malher, A., Boisson, J., and Touzé, C., 2016. "Design of a magnetic vibration absorber with tunable stiffnesses". Nonlinear Dynamics, 85(2), pp. 893-911.

[23] Lacarbonara, W., and Cetraro, M., 2011. "Flutter control of a lifting surface via viscohysteretic vibration absorbers". International Journal Aeronautical and Space Sciences, 12(4), pp. 331-345.

[24] Lee, B. H. K., Jiang, L., and Wong, Y., 1998. "Flutter of an airfoil with a cubic nonlinear restoring force". 39th AIAA/ASME/ASCE/AHS/ASC Structures, Structural Dynamics, and Materials Conf, 20-23 April, Long Beach, CA, 1998., pp. 237-257.

[25] Pettit, C. L., and Beran, P. S., 2003. "Effects of parametric uncertainty on airfoil limit cycle oscillation". Journal of Aircraft, 40(5), pp. 1004-1006.

[26] Bisplinghoff, R. L., and Ashley, H., 1962. Principles of aeroelasticity. John Wiley and Sons, Inc., New-York.

[27] Fung, Y. C., 2002. An introduction to the theory of aeroelasticity. Dover Publications, Inc., Mineola, New York.

[28] Lee, Y. S., Vakakis, A. F., Bergman, L. A., McFarland, D. M., and Kerschen, G., 2005. "Triggering mechansms of limit oscillations due to aeroelastic instability". Journal of Fluids and Structures, 21, pp. 485-529.

[29] Malher, A., Doaré, O., and Touzé, C., 2015. "Pseudoelastic shape memory alloys to mitigate the flutter instability: A numerical study". In Structural Nonlinear Dynamics and Diagnosis. Springer, pp. 353-365.

[30] Amandolèse, X., Michelin, S., and Choquel, M., 2013. "Low speed flutter and limit cycle oscillations of a two-degree-of-freedom flat plate in a wind tunnel". Journal of Fluids and Structures, 43, pp. 244-255.

[31] Dimitriadis, G., and Li, J., 2009. "Bifurcation behavior of airfoil undergoing stall flutter oscillations in low-speed wind tunnel". AIAA journal, 47(11), pp. 2577-2596.

[32] Kuznetsov, Y. A., 2013. Elements of applied bifurcation theory, Vol. 112. Springer, New York.

[33] Guckenheimer, J., and Holmes, P., 2013. Nonlinear oscillations, dynamical systems, and bifurcations of vector fields. Springer, New York.

[34] Manneville, P., 1995. "Dissipative structures and weak turbulence". Chaos-The Interplay Between Stochastic and Deterministic Behaviour, pp. 257-272. 
[35] Iooss, G., and Adelmeyer, M., 1998. Topics in bifurcation theory and applications. World Scientific, Singapore.

[36] Gai, G., and Timme, S., 2015. "Nonlinear reduced-order modelling for limit-cycle oscillation analysis". Nonlinear Dynamics, 84(2), pp. 991-1009.

[37] Touzé, C., Thomas, O., and Chaigne, A., 2004. "Hardening/softening behaviour in nonlinear oscillations of structural systems using non-linear normal modes". Journal of Sound and Vibration, 273(1), pp. 77-101.

[38] Doedel, E. J., Paffenroth, R. C., Champneys, A. R., Fairgrieve, T. F., Kuznetsov, Y. A., Sandstede, B., and Wang, X., 2002. Auto 2000: Continuation and bifurcation software for ordinary differential equations. Tech. rep., Concordia University.

[39] Woolston, D. S., 2012. "An investigation of effects of certain types of structural nonlinearities on wing and control surface flutter". Journal of the Aeronautical Sciences, 24(1), pp. 57-63. 


\section{Appendix A: Coefficients of Eq. (5)}

$$
\begin{aligned}
& a_{6}=r_{\alpha}^{2}-x_{\alpha}^{2} \\
& a_{5}=\left(\zeta_{h}+(1+\varepsilon) \zeta\right) r_{\alpha}^{2}+\left(\nu x_{\alpha}+b r_{\alpha}^{2}\right) \tilde{U}+\zeta_{\alpha}-x_{\alpha}^{2} \zeta+\left(2 x_{\alpha}+\lambda\right) \varepsilon \lambda \zeta, \\
& a_{4}=\left(\Omega^{2}+\zeta \zeta_{h}+(1+\varepsilon) \gamma+1\right) r_{\alpha}^{2}+\left(\beta \zeta_{\alpha}+\left(\nu x_{\alpha}+r_{\alpha}^{2} \beta\right) \zeta\right) \tilde{U}-\left(\beta x_{\alpha}+\nu\right) \tilde{U}^{2}+\left(\zeta_{h}+(1+\varepsilon) \zeta\right) \zeta_{\alpha} \\
& +\varepsilon \lambda^{2} \zeta \zeta_{h}-\gamma x_{\alpha}^{2}+\left(2 x_{\alpha}+\lambda\right) \varepsilon \gamma \lambda, \\
& a_{3}=\left(\zeta \Omega^{2}+(1+\gamma) \zeta_{h}+(1+\varepsilon) \zeta\right) r_{\alpha}^{2}-\left(\nu \zeta_{h}+\beta x_{\alpha} \zeta+(1+\varepsilon) \nu \zeta\right) \tilde{U}^{2}+\left(\beta \zeta \zeta_{\alpha}+\gamma \nu x_{\alpha}+r_{\alpha}^{2} \beta(1+\gamma)\right) \tilde{U} \\
& +\left(\zeta \zeta_{\alpha}+\varepsilon \gamma \lambda^{2}\right) \zeta_{h}+(1+\varepsilon) \gamma \zeta_{\alpha}+\left(\zeta_{\alpha}+\varepsilon \lambda^{2} \zeta\right) \Omega^{2}, \\
& a_{2}=\left((1+\gamma) \Omega^{2}+\zeta \zeta_{h}+(1+\varepsilon) \gamma\right) r_{\alpha}^{2}-\left(\nu \Omega^{2}+\nu \zeta \zeta_{h}+\beta \gamma x_{\alpha}+(1+\varepsilon) \gamma \nu\right) \tilde{U}^{2}+\beta\left(\gamma \zeta_{\alpha}+r_{\alpha}^{2} \zeta\right) \tilde{U} \\
& +\left(\zeta \zeta_{\alpha}+\varepsilon \gamma \lambda^{2}\right) \Omega^{2}+\gamma \zeta_{\alpha} \zeta_{h}, \\
& a_{1}=\left(\zeta \Omega^{2}+\gamma \zeta_{h}\right) r_{\alpha}^{2}-\nu\left(\zeta \Omega^{2}+\gamma \zeta_{h}\right) \tilde{U}^{2}+\beta \gamma r_{\alpha}^{2} \tilde{U}+\gamma \zeta_{\alpha} \Omega^{2}, \\
& a_{0}=\left(r_{\alpha}^{2}-\nu \tilde{U}^{2}\right) \gamma \Omega^{2} .
\end{aligned}
$$

\section{Appendix B: Coefficients of Eq. (6)}

The majority of the coefficients $a_{i j k}$ and $b_{i j k}$ from Eq. (6) are neglectable. The non-neglectable coefficients $a_{i j k}$ and $b_{i j k}$ are

$$
\begin{array}{llll}
a_{000}=0.328 & a_{100}=-0.629 & a_{200}=0.173 & a_{300}=0.294 \\
a_{010}=0.604 & a_{210}=0.165 & a_{030}=-0.124 & a_{001}=0.486 \\
a_{101}=-0.437 & a_{111}=-0.113 & a_{021}=-0.275 & a_{002}=0.278 \\
a_{102}=-0.254 & a_{003}=0.142 & a_{103}=-0.143 & \\
& & & \\
b_{000}=0.11 & b_{100}=0.0475 & b_{200}=-0.0701 & b_{300}=-0.174 \\
b_{010}=0.00307 & b_{210}=0.174 & b_{030}=-0.0329 & b_{001}=-0.00415 \\
b_{101}=-0.128 & b_{111}=0.106 & b_{021}=0.0164 & b_{002}=-0.081 \\
b_{102}=-0.0444 & b_{003}=-0.00191 & b_{103}=0.00259 . &
\end{array}
$$

\section{Appendix C: Coefficients of Eq. (10)}

The nonlinear coefficients of the cubic polynomial terms appearing in Eq. (10) are here detailed. Since the first transformation matrix has no simple analytic expression, the numerical values of the coefficients are given, which correspond to the airfoil parameters of the reference case given in Table 1 and to the optimal NLTVA linear parameters given in Table 2. 


$$
\begin{array}{ll}
c_{30}=0.047 \xi_{h}-0.064 \xi_{\alpha}-4.9 \xi & d_{30}=-0.01 \xi_{h}-0.43 \xi_{\alpha}-0.28 \xi \\
c_{21}=-0.054 \xi_{h}+0.16 \xi_{\alpha}+2.6 \xi & d_{21}=0.012 \xi_{h}+1.1 \xi_{\alpha}+0.15 \xi \\
c_{12}=0.021 \xi_{h}-0.13 \xi_{\alpha}-0.46 \xi & d_{12}=-4.5 .10^{-3} \xi_{h}-0.87 \xi_{\alpha}-0.026 \xi \\
c_{03}=-2.7 .10^{-3} \xi_{h}+0.036 \xi_{\alpha}+0.027 \xi & d_{03}=5.7 .10^{-4} \xi_{h}+0.24 \xi_{\alpha}+1.5 .10^{-3} \xi
\end{array}
$$

